\title{
Naturgefahren und Schäden für den Tourismus in den Alpen
}

\author{
Christian J. Nöthiger, Zürich, Rolf Bürki, St. Gallen, \\ Hans Elsasser, Zürich
}

\section{Einleitung und Zielsetzung}

«Tourism can...be significantly exposed to natural disasters, because of its attachment to high-risk areas with exotic scenery. The lure of snow-capped peaks brings the hazard of avalanches. Tropical beaches attract tourists to the potential paths of hurricanes...» (MURPhy \& BAYLEY 1989: 36).

Dieses Zitat und die jüngsten Ereignisse in Südostasien zeigen sehr klar, dass Bedrohungen durch Naturgefahren kein Hindernis für touristische Nutzungen darstellen. Im Gegenteil, oft ist in touristisch attraktiven Regionen die Gefahr von katastrophalen Naturereignissen besonders gross.

Der nachfolgende Artikel vermittelt zuerst einen generellen Überblick über den Forschungsstand zu «Naturgefahren und Tourismus». Anschliessend werden die für den Tourismus relevanten Naturgefahren und die Risiken im alpinen Berggebiet kurz vorgestellt. Bei den Schäden, die ein katastrophales Naturereignis anrichtet, muss zwischen direkten und indirekten unterschieden werden. Wie die Beispiele des Lawinenwinters 1999 und des Orkanes Lothar vom 26. Dezember 1999 zeigen, sind für die Tourismusbranche die indirekten Schäden oft um ein Mehrfaches grösser als die direkten. Den Abschluss bilden Ausführungen zu Massnahmen, die zur Minderung der Schäden und der Kosten ergriffen werden können.

Die Ausführungen stützen sich vor allem auf Untersuchungen, die über die Auswirkungen des Lawinenwinters 1999 auf den Tourismus in der Schweiz durchgeführt wurden. Da über die empirischen Resultate jener Untersuchungen bereits früher in der Geographica HelveTICA (NöTHIGER et al. 2002) berichtet wurde, liegt das Schwergewicht im vorliegenden Artikel auf allgemeineren Aussagen zum Thema «Naturgefahren und Tourismus». Davon ausgehend kann gezeigt werden, dass die Analyse konkreter Ereignisse eine Basis für die Herleitung allgemeiner Massnahmen bilden kann.

\section{Forschungsstand}

«...Relatively little systematic research has been carried out on disaster phenomena in tourism, the impacts of such events on the tourism industry and the responses of industry and relevant government agencies to cope with these impacts» (FAULKNER 2001: 136).
Diese treffende Aussage von FAULKNER bezieht sich nicht allein auf Naturkatastrophen, sondern auch auf katastrophale Ereignisse menschlichen Ursprungs, wie Flugzeugabstürze oder politische Krisen. Die gleiche Spannbreite von Ereignissen deckt auch die umfangreiche Arbeit zum «Krisenmanagement im Tourismus» von Glaesser (2001) ab. Für das stark praxisorientierte Handbuch «Tourist safety and security» der World Tourism Organization (WTO, 1996) gilt dasselbe.

Generell ist der Forschungsstand beim Zusammenhang zwischen Tourismus und katastrophalen Ereignissen menschlichen Ursprungs wesentlich besser als jener bei katastrophalen Naturereignissen. Dies gilt besonders für die Themenbereiche Terrorismus und politische Instabilität. Einen umfassenden und kommentierten Überblick über die entsprechende Literatur vor 1998 vermittelt SöNMEZ (1998). Als Beispiel für eine neuere Publikation zu «Tourismus und Terrorismus» sei auf den Artikel von Vorlaufer (2003) über Bali hingewiesen.

Bereits aus den siebziger und frühen achtziger Jahren des letzten Jahrhunderts existieren Arbeiten zu den Auswirkungen von katastrophalen Naturereignissen auf den Tourismus; so zu Lawinen und Hochwasser im französischen Val d'Isère (Hanns 1975), zu Muren und Lawinen im österreichischen Bundesland Salzburg (PIPPAN 1977) oder zum Ausbruch des Vulkans Mt. Usu in Nordjapan (Hirose 1982). Diese Untersuchungen gingen aber kaum über die Beschreibung einiger konkreter Ereignisse hinaus. Auch die Arbeit von MurPhy \& BAYLEY (1989) basierte auf konkreten Ereignissen, nämlich dem Ausbruch des Mount St. Helens (USA) vom Mai 1980 und den Waldbränden von East Kootenay in British Columbia (Canada) vom Juli 1985. In diesen Fällen wurden daraus allgemeine Schlussfolgerungen und Empfehlungen für die Tourismusbranche im Umgang mit katastrophalen Naturereignissen abgeleitet. In den nicht sehr zahlreichen Arbeiten aus den neunziger Jahren zu diesem Themenbereich wurde deshalb jeweils auf MurPhy \& Bayley Bezug genommen; so z.B. in Milo \& Yoder (1991), welche sich mit der Rolle von Reisejournalisten bei der Repositionierung von Tourismusdestinationen nach katastrophalen Naturereignissen beschäftigten, oder von Burby \& Wagner (1996), die den Schutz von Touristen vor tropischen Wirbelstürmen an der Küste des Golfs von Mexiko untersuchten. Am intensivsten beschäftigte sich in den neunziger Jahren der US-amerikanische Soziologe DrabeK mit den Zusammenhängen zwischen Tourismus und katastro- 


\begin{tabular}{|lll|}
\hline Häufigkeit in der Schweiz & Gebirgstypisch & Nicht gebirgstypisch \\
\hline Relativ häufig & Lawinen & Stürme \\
& Felsstürze & Unwetter \\
& Steinschlag & Hagel \\
& Dynamische Hochwasser & Statische Hochwasser \\
& Murgänge & \\
Relativ selten & Bergstürze & Verheerende Erdbeben \\
& Seeausbrüche & Meteoriteneinschläge \\
\hline
\end{tabular}

Tab. 1: Übersicht über die Naturgefahren in der Schweiz

Aperçu des dangers naturels en Suisse

Overview of natural hazards in Switzerland

phalen Naturereignissen. Seine Forschungsschwerpunkte lagen bei der Risikowahrnehmung und der Vorsorgeplanung touristischer Anbieter (DraBEK 1994) sowie beim Verhalten von evakuierten Touristen und deren Urteil über Massnahmen des Krisenmanagements (Drabek 1996). Dazu führte er Interviews mit 185 touristischen Anbietern in neun Gemeinden der USA durch und befragte 800 Touristen, welche von einem Erdbeben oder einem Hurrikan betroffen gewesen waren.

Am Beispiel des Erdbebens von Assisi im September 1997 wurde erstmals umfassend dargelegt, wie die finanziellen Auswirkungen eines katastrophalen Naturereignisses auf die Tourismusbranche ermittelt werden können (Mazzocchi \& Montini 2001). Das dabei verwendete Prinzip ist einfach: Die durch das katastrophale Naturereignis verursachte Abnahme der touristischen Frequenzen wird mit den durchschnittlichen Tagesausgaben eines Touristen multipliziert, was die Mindereinnahmen ergibt, die auf das Naturereignis zurückgeführt werden können. Dieses Vorgehen ist im Prinzip analog zu demjenigen, welches auch in touristischen Wertschöpfungsstudien (z.B. TsCHURTSCHENTHALER 1993; RÜTTER, GuHL \& MÜller 1996; KüPfER 2000) angewandt wird.

In der Schweiz existiert für die Zeit vor 1999 keine wissenschaftliche Literatur zum Thema «Naturgefahren und Tourismus». Mit dem Lawinenwinter 1999 und dem Sturm Lothar vom 26. Dezember 1999 änderte sich die Situation. In den beiden Ereignisanalysen zum Lawinenwinter (EIDGENÖsSISCHES INSTITUT FüR SCHNEE- UND LaWINENForschung (SLF) 2000) und zum Orkan Lothar (EIDGENÖsSISCHE ForSCHUNGSANStalt Für Wald, Schnee UNd Landschaft (WSL)
\& BUNdESAMT FÜR UMwelt, WALD UND LANDSCHAFT (BUWAL) 2001) wurden die Auswirkungen dieser beiden Naturereignisse auf die Tourismusbranche relativ breit erörtert. Vertiefte Studien beschäftigten sich mit den Auswirkungen des Lawinenwinters auf die (Tourismus-)Gemeinde Elm (NöTHIGER 2000), den Folgen der Ereignisse von 1999 auf die Schweizer Bergbahn- und Seilbahnunternehmen (NöTHIGER, BRÜNDL \& Ammann 2001) sowie den Konsequenzen des Lawinenwinters für die Tourismusbranche in Davos (Nöthiger \& Ammann 2001). Einen zusammenfassenden Überblick vermitteln Nöthiger et al. (2002); die detailreichste Darstellung mit einer Vielzahl von Literaturhinweisen findet sich in NöTHIGER (2003).

\section{Für den Tourismus relevante Naturgefahren im alpinen Berggebiet}

Grundsätzlich können alle katastrophalen Naturereignisse, welche in der Schweiz überhaupt denkbar sind, den alpinen Tourismus negativ beeinflussen. Nicht alle möglichen Ereignisse sind für die Tourismusbranche im Berggebiet allerdings gleichermassen relevant. Im Vordergrund stehen Ereignisse, welche einerseits relativ häufig und andererseits typisch für Gebirgsräume sind (Tab. 1). In erster Linie sind dazu gravitative Massenbewegungen wie Steinschlag und Felsstürze (Abb. 1), dynamische Hochwasser, Murgänge und insbesondere Lawinen zu zählen.

Katastrophale Naturereignisse, welche nicht als spezifisch alpin zu bezeichnen sind, weil sie das Umland des Alpenraums genauso betreffen wie das eigentliche Berggebiet (z.B. Stürme, Unwetter), haben im Vergleich dazu eine geringere Relevanz für die Touris- 


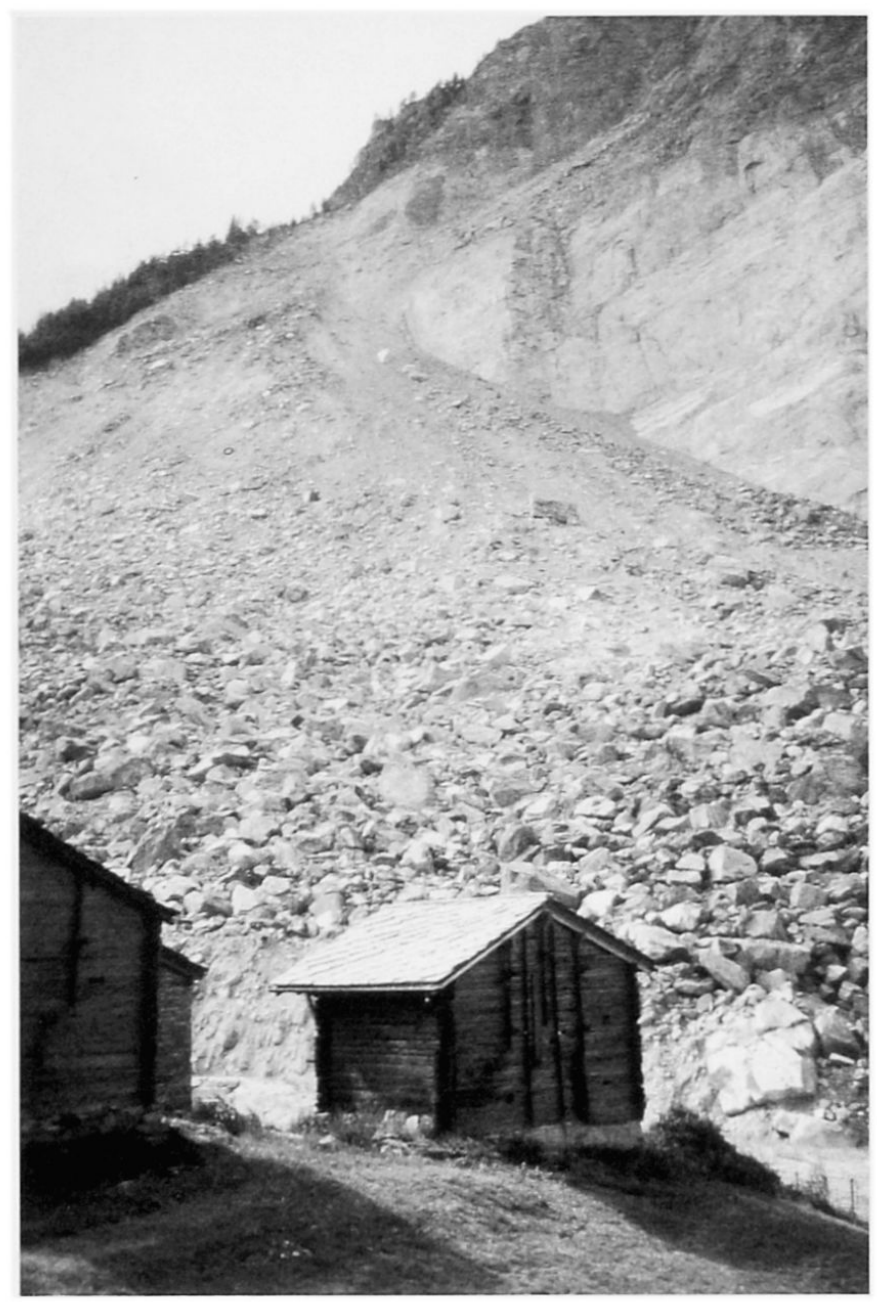

Abb. 1: Schuttkegel der Bergstürze von Randa (VS) vom 18. April und 19. Mai 1991. Diese Bergstürze betrafen insbesondere die Tourismusbranche in Zermatt, weil sie die Zufahrt auf Strasse und Schiene bis in den Sommer hinein beeinträchtigten.

Cône d'éboulement du 18 avril et du 19 mai 1991 à Randa (VS). Ces éboulements ont affecté particulièrement l'activité touristique de Zermatt, parce qu'ils ont empêché l'accès par la route et par le train jusqu'en été.

Debris of the rock avalanches of April 18 and May 19, 1991 in Randa (VS). These rock avalanches particularly affected tourism in Zermatt as they substantially impeded access by road and by rail well into the summer season.

Foto: C.J. NÖTHIGER, 23.7.1991

musbranche. Der Grund dafür liegt darin, dass solche Ereignisse potentiellen Gästen (aus dem Flachland) in Ablauf und Auswirkungen eher vertraut sind als gebirgstypische Ereignisse (vgl. NÖTHIGer 2003: 168 f.). Da sie jederzeit und überall auftreten können, ist kaum damit zu rechnen, dass sie beim Entscheid, ins Gebirge zu fahren, irgendeine Rolle spielen. Ebenfalls kaum relevant für die alpine Tourismuswirtschaft sind Ereignisse, deren Eintrittswahrscheinlichkeit derart gering ist, dass sie auch im Rahmen einer sehr langfristigen betriebswirtschaftlichen Planung nicht berücksichtigt werden können. Dazu können neben katastrophalen Erdbeben auch an sich gebirgstypische Ereignisse wie verheerende Bergstürze gezählt werden.

Unter einer Naturgefahr versteht man einen potenziell ablaufenden gefährlichen Prozess (KIENHOLz 1993: 9 und BUNDESAMT FÜr UMWELT, WALD UND LANDSCHAFT (BUWAL) 1998: 15). Ein gefährlicher Prozess ist ein Vorgang, der zu Schäden führen kann, sofern ihm verletzliche Objekte ausgesetzt sind. Ein Schaden ist die negativ bewertete Folge eines Ereignisses oder Vorgangs (BUNDESAMT FÜR UMWELT. WALD UND LANDSCHAFT (BUWAL) 1998: 6). Im Zusammenhang mit Naturgefahren kann das Risiko als

"Grösse und Wahrscheinlichkeit eines möglichen Schadens, der abhängig ist, einerseits von Ausmass und Eintretenswahrscheinlichkeit eines gefährlichen Prozesses an der Gefahrenstelle, anderseits von Wert und Präsenzwahrscheinlichkeit von potenziellen Schadenobjekten an derselben Gefahrenstelle"

verstanden werden (BUNDESAMT Für UMWELT, WALD UND LANDSCHAFT (BUWAL) 1998: 5).

\section{Direkte und indirekte Schäden}

Bei den Auswirkungen katastrophaler Naturereignisse stehen oft die direkten Schäden im Zentrum der Berichterstattung. Unter einem direkten Schaden versteht man einen Schaden, der direkt durch die Einwirkung des Schadenprozesses hervorgerufen wird. Verluste, die als Folge der durch ein Schadenereignis veränderten Bedingungen entstehen, werden als indirekte Schäden oder Folgeschäden bezeichnet (Bundesamt für Umwelt, Wald Und Landschaft (BUWAL) 1998: 6). Dabei wird davon ausgegangen, dass direkte Schäden monetarisiert werden können; direkte Schäden setzen ein potentiell gefährdetes Objekt voraus, dessen Wert beziffert werden kann. Direkte Schäden können deshalb auch als direkte Kosten bezeichnet werden. Die Entstehung direkter Schäden ist eng an den unmittelbaren Zeitpunkt eines Ereignisses gebunden. Für indirekte Auswirkungen ist dieser aber lediglich Ausgangspunkt und die Wirkungsdauer ist theoretisch zeitlich unbegrenzt. Direkte Schäden beschränken sich ferner auf den Prozessraum des katastrophalen Naturereignisses, indirekte Auswirkungen können im Extremfall global spürbar werden.

$\mathrm{Zu}$ den indirekten Schäden zählen alle Folgekosten eines katastrophalen Naturereignisses, die über die 


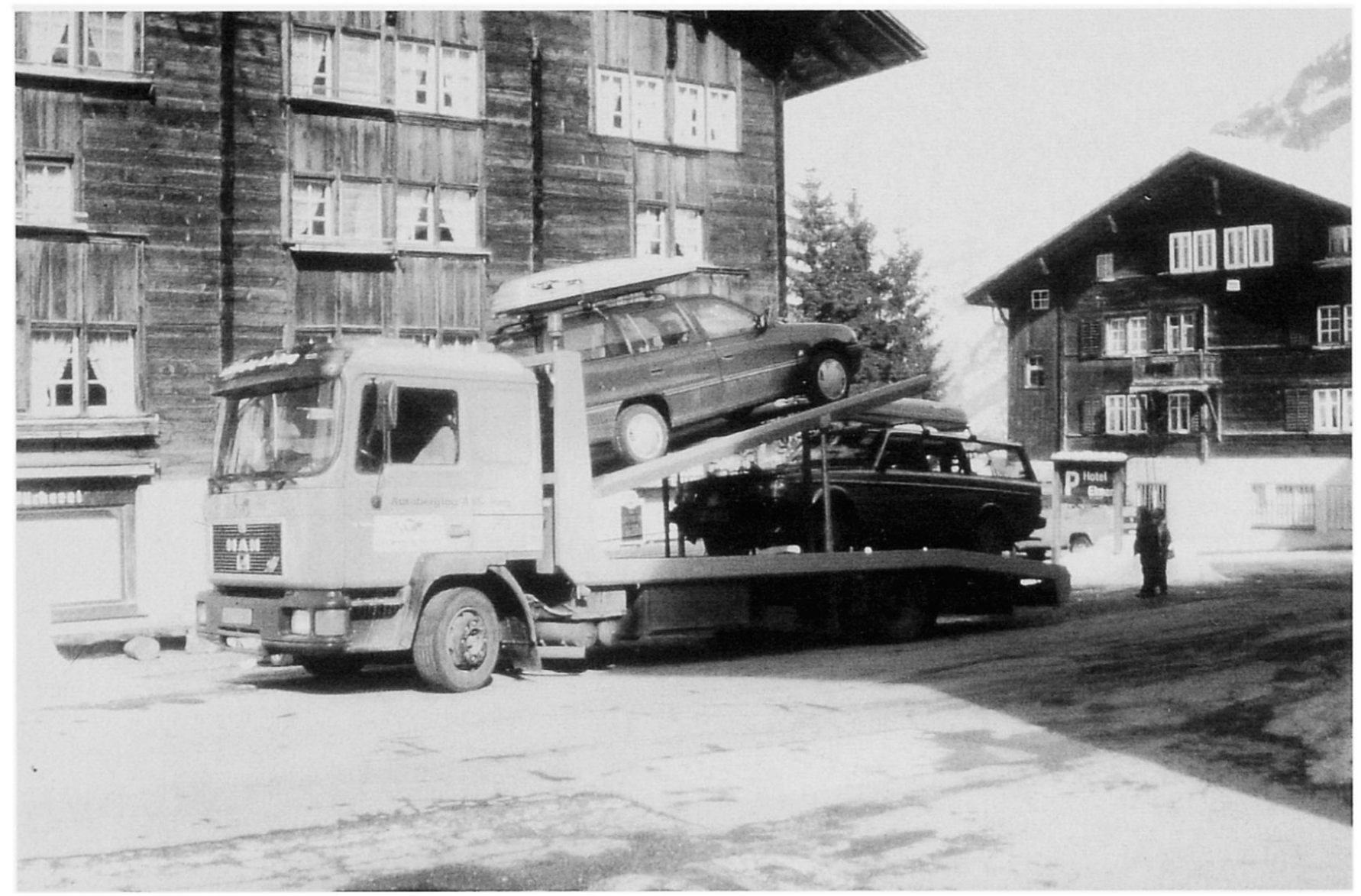

Abb. 2: Rückschaffung von während des Lawinenwinters 1999 in Elm (GL) zurückgelassenen Autos durch einen belgischen Abschleppdienst. Dieses Beispiel zeigt, wie auch weit vom Ereignisort entfernt angesiedelte Unternehmen von den Mehreinnahmen profitieren können, welche ein katastrophales Narurereignis verursacht.

Camion de dépannage belge ramassant des voitures abandonnées pendant les avalanches de l'hiver 1999 à Elm $(G L)$. Cet exemple montre que même des entreprises situées très loin du lieu d'une catastrophe naturelle peuvent profiter des recettes supplémentaires causées par cet évènement.

Belgian breakdown lorry collecting cars which had been left behind in Elm $(G L)$ during the avalanche winter of 1999. This exemplifies the profit to be gained from natural disasters even by firms situated a long way from the actual site of the disaster.

Foto: C.J. NÖTHIGER, 10.3.1999

Räumungs- und Instandsetzungskosten von direkten Schäden hinausgehen. Im Vordergrund stehen dabei die wirtschaftlichen Folgekosten des Ereignisses. Es handelt sich dabei um Mindereinnahmen, die durch das katastrophale Naturereignis über Produktionsausfälle oder -erschwernisse, den Verlust an Marktanteilen u.ä. ausgelöst werden. Indirekte Kosten können nicht nur in der vom katastrophalen Naturereignis direkt betroffenen Region auftreten, sondern auch weiter entfernte Betriebe können von Zulieferungen zerstörter Betriebe abhängig sein. Anderseits ist denkbar, dass bestimmte Firmen neue Aufträge erhalten, die von zerstörten Betrieben nicht mehr ausgeführt werden können, d.h. diese Firmen kommen durch das Ereig- nis zu Mehreinnahmen (Abb. 2). Touristen können sich für eine andere, als sicherer betrachtete Tourismusdestination entscheiden und dort spürbare Zusatzeinnahmen verursachen. Es besteht allerdings auch die Möglichkeit, dass eine Region im Nachhinein von einem katastrophalen Naturereignis profitiert. Ein solches Ereignis weckt oft grosses öffentliches Interesse und die betroffene Region wird von "Sensationstouristen» besucht, die dann dort auch gewisse Ausgaben im Gastgewerbe und Detailhandel tätigen.

Katastrophale Naturereignisse können aber im Nachhinein auch bewusst touristisch in Wert gesetzt werden. Das klassische Beispiel sind die durch den Ausbruch 


\section{Direkte Schäden}

\begin{tabular}{|lccc|} 
Schadensbereich & Geschätzte Kosten & Schadensbereich & Geschätzte Kosten \\
\hline Gebäude und Fahrhabe & 252 Mio. SFr. & Übernachtungen & 42 Mio. SFr. \\
Strassen & 63 Mio. SFr. & Gastronomie & 109 Mio. SFr. \\
Schiene & 11 Mio. SFr. & Detailhandel & 46 Mio. SFr. \\
Bergbahnen/Skilifte & 17 Mio. SFr. & Bergbahnen/Skilifte & 83 Mio. SFr. \\
Schutzbauten & 9 Mio. SFr. & Übriges & Mio. SFr. \\
Elektrizitätsnetz & 27 Mio. SFr. & & \\
Wald & 46 Mio. SFr. & & 302 Mio. SFr. \\
Landwirtschaftsflächen & 14 Mio. SFr. & & Total \\
\hline Total & $\mathbf{4 9 3}$ Mio. SFr. & & \\
\hline
\end{tabular}

Tab. 2: Übersicht über die Kosten von direkten und indirekten Lawinen- und Schneedruckschäden des Lawinenwinters 1999 in der Schweiz

Aperçu des coûts directs et indirects provoqués par les avalanches de l'hiver 1999 en Suisse

Overview of the costs of direct and indirect damages caused by avalanches during the 1999 winter in Switzerland

Quelle: Eidgenössisches Institut für SchneE- Und LaWinenforschung 2000: 265; Nöthiger 2003: 133

des Vesuvs zerstörten Römerstädte Pompeji und Herculaneum. Ein aktuelleres Beispiel ist das Mount St. Helens National Volcanic Monument in den USA, welches zwei Jahre nach der verheerenden Eruption vom 18. Mai 1980 geschaffen wurde (Murphy \& Bayley 1989).

Die direkten Schäden des Lawinenwinters 1999 in der Schweiz betrugen 439 Mio. SFr. (Tab. 2). Davon entfielen rund 30 Mio. SFr. oder knapp 7\% auf die Tourismusbranche (NöTHIGER 2003: 39 ff.). Die indirekten Schäden, d.h. die Mindereinnahmen der Tourismusbranche wurden von NöTHIGER (2003: 133) auf 302 Mio. SFr. geschätzt, dies bedeutet, dass die indirekten Schäden im Tourismus rund zehnmal so hoch waren, wie die direkten.

Die direkten Schäden des Orkans Lothar vom 26. Dezember 1999 betrugen insgesamt 1520 Mio. SFr., davon entfielen $8 \mathrm{Mio}$. SFr. auf Bergbahnen und Skilifte (NöTHIGER 2003: 166). In welchem Ausmass gastgewerblich genutzte Gebäude betroffen waren, ist unbekannt. Der hohe Gesamtschaden des Orkans Lothar rührt daher, dass die hauptsächlichen Schäden im dicht besiedelten Mittelland und nicht im alpinen Raum auftraten. Bei den Bergbahnen und Skiliften verursachte der Orkan Lothar im Vergleich zum Lawinenwinter mehr Schadensfälle, die durchschnittlichen Schadenskosten pro Anlage und auch die Gesamtschadensumme waren aber geringer. Die durch den Orkan Lothar verursachten indirekten Kosten, d.h. die Mindereinnahmen für die Tourismusbranche in der Schweizer Bergzone, wurden auf 113 Mio. SFr. geschätzt (NöTHIGER 2003: 173). Auch bei diesem katastrophalen Naturereignis waren für die Tourismusbranche die indirekten Kosten wesentlich höher als die direkten.

Ein wesentlicher Unterschied zwischen direkten und indirekten Schäden besteht bei der Frage nach der Entschädigung. Direkte Kosten sind grösstenteils durch Versicherungen oder die öffentliche Hand abgedeckt. Ganz anders sieht das Bild bei den indirekten Kosten aus, die zum überwiegenden Teil von den betroffenen Betrieben und Haushalten getragen werden müssen. Das Fallbeispiel Elm (NöTHIGER 2000) zeigt, dass die direkten Kosten des Lawinenwinters 1999 zu 6\%, die indirekten zu 93\% und die Gesamtkosten zu 40\% von den Betrieben und Haushalten übernommen werden mussten. 


\section{Massnahmen}

Sämtliche Massnahmen, die direkte Schäden von katastrophalen Naturereignissen verhindern sollen, sind zugleich auch präventive Massnahmen gegenüber indirekten Schäden. Bei der Frage, ob die Kosten einer zusätzlichen Massnahme vom Umfang der damit verhinderten Schadenskosten aufgewogen werden oder nicht, gilt es unbedingt auch die indirekten Schäden zu berücksichtigen. Geht man von einem zu tiefen Nutzen aus, wird eine Massnahme möglicherweise verworfen, obwohl sie sich unter Berücksichtigung der indirekten Kosten lohnen würde. Der Grund für den Nichteinbezug indirekter Schäden sind die Schwierigkeiten bei deren Ermittlung.

Bei der Vorbereitung von Schutzmassnahmen gegen direkte Schäden von katastrophalen Naturereignissen kommt der Tourismusbranche nur eine geringe Bedeutung zu (WiLhelm 1996: 234 f.). Eine Ausnahme bilden Bergbahn- und Skiliftunternehmen, die Fachpersonal für die Beurteilung der Lawinengefahr in einem Wintersportgebiet benötigen, das künstliche Auslösungen von Lawinen und allenfalls Pistensperrungen vornehmen kann. Die WorLd Tourism OrGaNIZATION (WTO, 1996: 90) empfiehlt Tourismusbetrieben als präventive Massnahme die Einsichtnahme in die Notfallpläne des Zivilschutzes. Damit soll sichergestellt werden, dass in diesen Plänen die Anwesenheit einer allenfalls grösseren Zahl von Touristen berücksichtigt wird.

Neben der Prävention spielen aber auch Massnahmen während und nach einem Ereignis eine wichtige Rolle, um indirekte Schäden gering zu halten. In einer Umfrage bei den Schweizer Bergbahn- und Skiliftunternehmen nach dem Lawinenwinter 1999, die danach fragte, welche besonderen Massnahmen von diesen Unternehmen aufgrund der gemachten Erfahrungen in Zukunft ergriffen werden sollten, um die indirekten Kosten von Naturereignissen zu senken, wurden in erster Linie eine aktivere Medienarbeit (externe Kommunikation) und eine bessere Kundeninformation (interne Kommunikation) genannt (Nöthiger 2003: 147). Der externen und internen Kommunikation der Tourismusunternehmen und -organisationen kommt somit eine zentrale Bedeutung zu bei der Bewältigung von Naturkatastrophen. Die folgenden Empfehlungen der WTO zur externen Kommunikation dürften jedoch nicht nur für den Bereich des Tourismus Gültigkeit besitzen: «Sei schnell, sei ehrlich und sachlich, geh auf die Medien ein, sei vorbereitet" (World Tourism Organization (WTO) 1996: 29 f.). Im Vergleich zur externen Kommunikation ist allerdings der internen Kommunikation, d.h. der Kommunikation vor Ort, Priorität einzuräumen, weil sie sich an Personen richtet, welche durch ein katastrophales Naturereignis potenziell gefährdet sind.

Die wichtigste Massnahme nach dem Ende der akuten Phase eines katastrophalen Naturereignisses ist die Wiederinbetriebnahme bzw. Wiederherstellung der touristischen Infrastruktur. Tourismusbetriebe besitzen die Möglichkeit, Versicherungen gegen die finanziellen Folgen von katastrophalen Naturereignissen abzuschliessen. Bei den direkten Kosten sind vor allem die Gebäudeversicherungen zu nennen. Was die indirekten Kosten betrifft, sind Betriebsunterbrechungsversicherungen sowie Versicherungen gegen Rückwirkungsschäden zu erwähnen. Der Versicherungsfall muss allerdings an «einen versicherbaren Sachschaden oder ein anderes klar erkenn- und abgrenzbares Ereignis» (STEINER 1970: 53) gekoppelt sein. Als Ursache für Rückwirkungsschäden könnte in der Police z.B. auch die Blockierung einer Zufahrtsstrasse als solches Ereignis definiert werden. Bei Ereignissen, deren Eintretenswahrscheinlichkeit schlecht bekannt ist, müssen die Versicherungsgesellschaften allerdings relativ hohe Prämien festlegen. Deswegen sind Versicherungen gegen Rückwirkungsschäden in der Tourismusbranche kaum verbreitet. Dies wiederum erklärt, weshalb die indirekten Kosten von katastrophalen Naturereignissen zum überwiegenden Teil von den Betroffenen selbst getragen werden müssen.

Mit der Wiederinstandsetzung der Infrastruktur nach einer Naturkatastrophe ist die Situation für die Tourismusbranche noch nicht vollständig bereinigt, denn

«...the recovery of destinations usually takes longer than the period required for the restoration of services to normalcy" (FAULKNER 2001: 142).

Sehr wichtig ist, dass nach der Bewältigung des Ereignisses rasch erste Gäste zurückkehren. Die Gründe dafür nennen MurPhy \& BAYLEY (1989: 39):

"Such visitors will bear witness to the re-established safety and attractiveness of the area and will bring new revenue to assist in reconstruction. Successfull visits can reinforce the fact that tourism has survived and that recovery is under way.»

Bei der Unterstützung dieses Prozesses kommt auch in dieser Phase wieder der externen und internen Kommunikation vorrangige Bedeutung zu. Ein Hauptproblem der Öffentlichkeitsarbeit nach einem katastrophalen Naturereignis besteht jedoch darin, dass ein bewältigtes Ereignis für die Medien keinen hohen Nachrichtenwert besitzt. Gerade für den Tourismus ist jedoch die Botschaft, dass alles wieder «normal» sei und dass die Gäste wieder kommen könnten, sehr wichtig. Je rascher sich die Situation normalisiert, desto geringer sind indirekte Schäden, aber diese Normalisierung muss erfolgreich kommuniziert werden. 


\section{Literatur}

Burby, R.J. \& F. WaGner (1996): Protecting tourists from death and injury in costal storms. - In: Disasters. The international journal of disaster studies amd practice 20,1:49-60.

BUNDESAMT FÜR UMWELT, WALD UND LANDSCHAFT (BUWAL) (1998): Begriffsdefinitionen zu den Themen Geomorphologie, Naturgefahren, Forstwesen, Sicherheit und Risiko. - Arbeitspapier, Bern: BUWAL/ Eidgenössische Forstdirektion.

DRABEK, T.E. (1994): Risk perception of tourist business managers. - In: The environmental professional. The official journal of the national association of environmental professionals (NAEP) 16, 4: 327-341.

DRABEK, T.E. (1996): Disaster evacuation behaviour. Tourists and other transients $-=$ Program on environment and behaviour. Monograph No 58. Institute of behavioural science, natural hazards research and applications information centre, University of Colorado, Boulder.

EIDGENÖSSISCHE ForsChUNGSANSTALT FÜR WALD, SCHNEE UND LANDSCHAFT (WSL) \& BUNDESAMT FÜr UMWELT, WALD UND LANDSCHAFT (BUWAL) (2001): Lothar. Der Orkan 1999. Ereignisanalyse. - Birmensdorf/Bern: WSL/BUWAL.

EIDGENÖSSISCHES INSTITUT FÜR SCHNEE- UND LAWINENFORSCHUNG (SLF) (2000): Der Lawinenwinter 1999. Ereignisanalyse. - Davos: SLF.

FAULKNER, B. (2001): Towards a framework for tourism disaster management. - In: Tourism management 22: 135-147.

Glaesser, D. (2001): Krisenmanagement im Tourismus. - Frankfurt a.M.: Peter Lang.

Hanns, C. (1975): Die Lawinen- und Überschwemmungsschäden in der Wintersportstation Val d'Isère (Hochtarantaise). Folgen einer physiogeographische Gegebenheiten nicht berücksichtigenden Siedlungserweiterung. - In: Geographische Rundschau 9/75: 390-400.

Hirose, H. (1982): Volcanic eruption in northern Japan. - Disasters. The international journal of disaster studies and practice 6, 2: 89-91.

Kienholz, H. (1993): Naturgefahren. Naturrisiken im Gebirge. - In: Eidgenössische Forschungsanstalt Für Wald, SchneE UNd Landschaft (WSL) (Hrsg.): Naturgefahren. - Publikation zur Tagung «Forum für Wissen» vom 28. Januar 1993 an der WSL, Birmensdorf: 7-21.

KüPFER, I. (2000): Die regionalwirtschaftliche Bedeutung des Nationalparktourismus. Untersucht am Beispiel des Schweizerischen Nationalparks. - = Nationalpark-Forschung in der Schweiz 90, Zernez.

Mazzocchi, M. \& A. Montini (2001): Earthquake effects on tourism in central Italy. - In: Annals of tourism research 28, 4: 1031-1046.

MiLo, K.J. \& S.L. Yoder (1991): Recovery from natural disaster. Travel writers and tourist destinations. -
In: Journal of travel research. A quarterly publication of the Travel and Tourism Research Association 30,1: 36-39.

MuRPhy, P.E. \& R. Bailey (1989): Tourism and disaster planning. - In: Geographical Review 79, 1: 36-46.

NöTHIGER, C.J. (2000): Der Lawinenwinter 1999. Fallstudie Elm (Kanton Glarus). Indirekte Auswirkungen auf die lokale Wirtschaft. - Davos: Eidgenössisches Institut für Schnee- und Lawinenforschung.

NöTHIGER, C.J. (2003): Naturgefahren und Tourismus in den Alpen. Untersucht am Lawinenwinter 1999 in der Schweiz. - Davos: Eidgenössisches Institut für Schneeund Lawinenforschung.

Nöthiger, C.J. \& W.J. Ammann (2001): Die Auswirkungen des Lawinenwinters 1999 auf die Betriebe und die Feriengäste in der Gemeinde Davos (Kanton Graubünden). - = Interner Bericht Nr. 744 des Eidgenössischen Instituts für Schnee- und Lawinenforschung, Davos.

Nöthiger, C.J., Bründl, M. \& W.J. Ammann (2001): Die Auswirkungen der Naturereignisse 1999 auf die Bergbahn- und Skiliftunternehmen der Schweiz. - In: Zeitschrift für Fremdenverkehr 1/01: 23-32.

Nöthiger, C.J. et al. (2002): Indirekte Auswirkungen von Naturgefahren auf den Tourismus. Das Beispiel des Lawinenwinters 1999 in der Schweiz. - Geographica Helvetica 2/02: 91-108.

PIPPAN, T. (1977): Der Einfluss von katastrophalen Wetterereignissen auf den Saison-Fremdenverkehr und die behördlichen Kontrollmassnahmen am Beispiel des Landes Salzburg. - In: Beiträge zur geographischen Methode und Landeskunde (Festgabe für Gudrun Höhl). - = Mannheimer Geographische Arbeiten 1, Mannheim: 401-315.

RütTer, H., Guhl, D. \& H.R. Müller (1996): Wertschöpfer Tourismus. Ein Leitfaden zur Berechnung der touristischen Gesamtnachfrage, Wertschöpfung und Beschäftigung in 13 pragmatischen Schritten. Bern: Forschungsinstitut für Freizeit und Tourismus der Universität Bern (FIF).

SönmEZ, S.F. (1998): Tourism, terrorism and political instability. - In: Annals of tourism research 259, 2: 416-456.

Steiner, P. (1970): Die Betriebsunterbrechungs-Versicherung in der Schweiz. - = Veröffentlichungen des Instituts für Versicherungswirtschaft an der Hochschule St. Gallen HSG 15, Zürich: Polygraphischer Verlag.

Tschurtschenthaler, P. (1993): Methoden zur Berechnung der Wertschöpfung im Tourismus. - In: HaEDRICH, G. et al. (Hrsg.): Tourismus-Management. Tourismus-Marketing und Fremdenverkehrsplanung. - 2., völlig neu bearbeitete und wesentlich erweiterte Auflage, Berlin, New York: Walter de Gruyter: 214-241.

VORLAufer, K. (2003): Bali. Tourismus und Terror im «Inselparadies». - In: Geographische Rundschau 3/03: 50-55. 
WiLhelM, C. (1996): Wirtschaftlichkeit im Lawinenschutz. Methodik und Erhebungen zur Beurteilung von Schutzmassnahmen mittels quantitativer Risikoanalyse und ökonomischer Bewertung. - Davos: Eidgenössisches Institut für Schnee- und Lawinenforschung.

World Tourism Organization (WTO) (1996): Tourist safety and security. Practical measures for destinations. - Madrid: WTO.

\section{Zusammenfassung: Naturgefahren und Schäden für den Tourismus in den Alpen}

Der Tourismus ist, nicht nur in den Alpen, in erheblichem Ausmass Naturgefahren ausgesetzt. Bis anhin ist jedoch verhältnismässig wenig systematische Forschung zum Problemkreis «Naturgefahren und Tourismus» durchgeführt worden, weshalb über die Auswirkungen katastrophaler Naturereignisse auf die Tourismusbranche und auf den Umgang der Branche mit diesen Auswirkungen nur wenige detaillierte und gesicherte Erkenntnisse vorliegen. In der Schweiz setzte die Auseinandersetzung mit diesen Problemen erst nach dem Lawinenwinter 1999 und dem Orkan Lothar vom 26. Dezember 1999 ein. Im Alpenraum bilden gravitative Massenbewegungen und insbesondere Lawinen die wichtigsten Naturgefahren für den Tourismus. Bei den durch katastrophale Naturereignisse verursachten Schäden muss zwischen direkten und indirekten Schäden unterschieden werden. Der Lawinenwinter 1999 verursachte in der Schweiz im Tourismus direkte Schäden in der Höhe von 30 Mio. SFr. und indirekte in der Höhe von 302 Mio. SFr. Die direkten Schäden des Orkans Lothar betrugen bei den Bergbahnen und Skiliften 8 Mio. SFr., die indirekten Schäden für den Tourismus insgesamt wurden auf $113 \mathrm{Mio}$. SFr. geschätzt. Damit wird deutlich, dass für die Tourismuswirtschaft die indirekten Schäden wesentlich bedeutender sind als die direkten. Je rascher es gelingt, die Situation nach einem katastrophalen Naturereignis zu normalisieren, desto geringer sind die indirekten Kosten für die touristischen Unternehmen. Diese Normalisierung muss aber erfolgreich kommuniziert werden. Die Schwierigkeit dabei besteht darin, dass eine Naturkatastrophe ein Medienereignis darstellt, die Normalisierung jedoch nur auf geringes Interesse bei den Medien stösst. Bei der Bewältigung der Auswirkungen von solchen Ereignissen kommt deshalb der Öffentlichkeitsarbeit von Tourismusunternehmen und -organisationen eine zentrale Bedeutung zu.

\section{Résumé: Les conséquences des dangers naturels sur l'activité touristique dans les Alpes}

Le tourisme n'est pas uniquement exposé de façon notoire aux dangers naturels dans les Alpes. Mais comme la recherche ne s'est penchée jusqu'à présent que relativement peu, de manière systématique, sur la thématique portant sur «les dangers naturels et le tourisme», nous ne disposons que peu de données détaillées et constatations scientifiques concernant les répercussions catastrophiques d'événements naturels sur le secteur du tourisme et les rapports de ce secteur avec ces répercussions. En Suisse, la prise en considération de ces problèmes n'a commencé qu'après les avalanches de l'hiver 1999 et l'ouragan Lothar du 26 décembre 1999. Dans l'arc alpin, les mouvements de masse gravitaires et particulièrement les avalanches constituent les dangers naturels les plus importants pour le tourisme. En ce qui concerne les effets négatifs des catastrophes naturelles, il faut distinguer les dégâts directs des dégâts indirects. Les avalanches de l'hiver 1999 ont causé en Suisse, dans la branche touristique, des coûts directs de 30 millions et des coûts indirects de 302 millions CHF. Les coûts directs de l'ouragan Lothar pour les sociétés de remontées mécaniques suisses se sont élevés à 8 millions $\mathrm{CHF}$, tandis que les coûts indirects pour la branche touristique entière ont vraisemblablement atteint environ 113 millions CHF. On peut en déduire que pour la branche touristique les effets indirects d'une catastrophe naturelle sont beaucoup plus importants que les effets directs. Plus vite on réussit à normaliser la situation après une catastrophe naturelle, plus faibles seront les coûts indirects pour les entreprises touristiques. Cette normalisation doit, toutefois, être communiquée avec succès. La difficulté réside dans le fait qu'une catastrophe naturelle est un événement médiatique, tandis que la normalisation de la situation n'intéresse que très peu les médias. C'est pourquoi l'action publique des entreprises et des organisations touristiques joue un rôle crucial dans la maîtrise des effets de tels événements.

\section{Summary: Natural Hazards and Damages to Tourism in the Alps}

The tourist industry has always been highly threatened by natural disasters, be it in the Alps or elsewhere. However, relatively little systematic research has been carried out on the subject of «natural hazards and tourism» resulting in a lack of detailed and accepted knowledge on the effects of natural disasters on tourism and on the response of the tourist industry to such events. In Switzerland, science did not deal with these problems at all before the avalanche winter of 1999 and the winter storm Lothar on December 26, 1999. Rapid mass-wasting processes and avalanches in particular can be considered to be the most important natural hazards for tourism. Negative effects caused by natural disasters can be divided into direct and indirect damages. Direct costs of the avalanche winter of 1999 for the Swiss tourist industry added up to 30 Mio. Swiss Francs, whereas indirect costs have been estimated to amount to 302 Mio. Swiss Francs. The winter storm Lothar caused the Swiss cable car companies direct costs of 8 Mio. Swiss Francs; the indirect costs for 
the whole Swiss tourist industry added up to approximately 113 Mio. Swiss Francs. This illustrates that indirect damages are of considerably higher relevance to the tourist industry than direct damages. The sooner the pre-disaster situation can be re-established, the smaller the indirect costs for tourism will be. Successful recovery has to be communicated though. The biggest problem while doing so is that whereas natural disasters are of exceptional interest for the media, successful recovery is not. Therefore, public relations should be a central issue to any tourist firm or organisation dealing with the effects of such events.

Dr. Christian J. Nöthiger, Rütschistrasse 27, CH-8037 Zürich.

e-mail: ch.noethiger@gmx.ch

Dr. Rolf Bürki, Pädagogische Hochschule St. Gallen, Notkerstrasse 27, CH-9000 St. Gallen.

e-mail: rbuerki@bluewin.ch

Prof. Dr. Hans Elsasser, Abteilung Wirtschaftsgeographie, Geographisches Institut der Universität Zürich, Winterthurerstrasse 190, CH-8057 Zürich.

e-mail: elsasser@geo.unizh.ch

Manuskripteingang/received/manuscrit entré le

17.6.2004

Annahme zum Druck/accepted for publication/accepté pour l'impression: 8.3 .2005 